\title{
Serpentes da Bacia Petrolífera de Urucu, Município de Coari, Amazonas, Brasil
}

\author{
Ana Lúcia da Costa PRUDENTE ${ }^{1}$; Gleomar Fabiano MASCHIO²; Maria Cristina dos SANTOS-COSTA; \\ Darlan Tavares FEITOSA ${ }^{4}$
}

\section{RESUMO}

Um inventário estruturado de serpentes foi realizado na Base Operacional Geólogo Pedro de Moura (BOGPM), localizada na Bacia Petrolífera de Urucu, Município de Coari, Amazonas, nos anos de 2003, 2004 e 2007. Nas quatro expediçóes realizadas ( 51 dias de coleta) foram registradas 47 espécies de serpentes, pertencentes a sete famílias e 33 gêneros. Foram utilizados quatro métodos complementares de amostragem de serpentes: armadilha de interceptaçáo e queda, encontros ocasionais, procura limitada por tempo a pé e procura limitada por tempo de carro. Das 47 espécies coletadas, Liophis reginae $(\mathrm{n}=14)$, Philodryas viridissima $(\mathrm{n}=9)$, Philodryas boulengeri $(\mathrm{n}=7)$ e Oxybelis fulgidus $(\mathrm{n}=7)$ foram as mais abundantes em toda região. O maior número de espécies e espécimes foi registrado pela procura limitada por tempo de carro (52,8\%). Estudos anteriores indicam que as localidades ao sul do Rio Amazonas (como região Leste do Pará, Usina Hidrelétrica de Tucuruí, Estado do Pará, e de Samuel, Estado de Rondônia) apresentam maior riqueza quando comparadas às regiôes ao norte do Amazonas (como Município de Manaus, Reserva do INPA-WWF e Usina Hidrelétrica de Balbina, Estado do Amazonas). Desta forma, é possível inferir que o levantamento das serpentes da região de Urucu ainda náo esteja completo, sendo necessário um maior esforço de coleta para que novos registros sejam adicionados para a área.

PALAVRAS-CHAVE : Amazônia ocidental, Squamata, diversidade, inventário estruturado.

\section{Snakes of the Urucu Petrol Basin, Municipality of Coari, Amazonas, Brasil}

\begin{abstract}
A structured snake inventory was carried out at the Base Operacional Geólogo Pedro de Moura (BOGPM), located at the Urucu Petrol Basin, Municipality of Coari, Amazonas, during 2003, 2004 and 2007. Throughout four sampling expeditions (51 collection days), seven species of snakes, belonging to seven families and 33 genera, were recorded. Four complementary sampling methods were used: pitfall traps with drift fence, occasional encounters, time-limited search on foot and time-limited search by car. Of the 47 collected species Liophis reginae $(\mathrm{n}=14)$, Philodryas viridissima $(\mathrm{n}=9)$, Philodryas boulengeri $(\mathrm{n}=7)$ and Oxybelis fulgidus $(\mathrm{n}=7)$ were the most abundant in the region. The highest number of species and specimens was obtained by the time-limited search by car $(\mathrm{n}=52.8 \%)$. Previous studies indicate that localities south of the Amazonas river (such as the eastern region of Pará and Tucuruí, state of Para, and Samuel Hydroelectric Plants, state of Rondônia) present a greater richness when compared to those north of the Amazonas river (such as the mucipality of Manaus, INPA-WWF Reserve and Balbina Hydroelectric Plant, state of Amazonas).Thus, it is possible to infer that the snake inventory in Urucu region is not yet complete, requiring more sampling efforts so that new records can be obtained for the area.
\end{abstract}

KEY WORDS : Ocidental Amazon, Squamata, diversity, structured inventory

\footnotetext{
1 Museu Paraense Emílio Goeldi, E-mail: prudente@museu-goeldi.br

2 Museu Paraense Emílio Goeldi, E-mail: gleomarmaschio@yahoo.com.br

3 Universidade Federal do Pará, E-mail: mcsc@ufpa.br

${ }^{4}$ Museu Paraense Emílio Goeldi, E-mail: dtfeitosa@yahoo.com.br
} 


\section{INTRODUÇÃ̃O}

As florestas tropicais úmidas são conhecidas por apresentar a maior diversidade de serpentes em relação aos demais biomas. Acredita-se que os processos de especiação que implicaram na grande riqueza atual de espécies na Amazônia seja resultado das mudanças no clima e vegetação ocorridas durante o Pleistoceno (Cracraft 1985). Fatores ecológicos também podem influenciar a riqueza de espécies em uma comunidade de serpentes, como exemplo, a estrutura da vegetação, presença de grandes corpos d'água e disponibilidade de presas (Vitt e Vangilder 1983; Cadle e Greene 1993; Martins e Oliveira 1998).

Analisando o padráo de diversidade de Squamata Neotropicais de 16 localidades, Silva e Sites (1995) definiram três grupos com padrôes de distribuição distintos na América Central, na Amazônia e nas regiôes mais secas da América do Sul. Na Amazônia brasileira, Silva e Sites (1995) comparando a fauna de serpentes (149 espécies, sensu Ávila-Pires et al. 2007), revelaram a formação de dois grupos limitados pelo Rio Amazonas, estando ao norte três localidades no Estado do Amazonas (Manaus, Balbina e Reserva da WWF) e ao sul duas regiôes localizadas no Estado do Pará (Leste do Pará e UHE Tucuruí) e uma em Rondônia (UHE Samuel).

Localizada ao sul do Rio Amazonas, a Bacia Petrolífera de Urucu, Município de Coari, apresenta uma matriz de floresta tropical úmida bem preservada, embora seja uma área de intensa atividade de exploração de petróleo e gás natural, com a presença de inúmeras clareiras e poços. Como parte do projeto de "Dinâmica de Clareiras Sob Impacto da Exploração Petrolífera, Rede CTPETRO Amazônia", foi realizado um inventário estruturado da fauna de serpentes, cujos resultados apresentam o registro de espécies de serpentes da região, incluindo comentários taxonômicos.

\section{MATERIAL E MÉTODOS}

\section{ÁREA DE ESTUDO}

O estudo foi realizado na Base Operacional Geólogo Pedro de Moura (BOGPM) (453’7,33”S; 65²0’59,99”W), área de 514.000 ha, localizada na Província Petrolífera de Urucu, Município de Coari, Amazonas (Figura 1). O clima da regiáo de Urucu apresenta um ciclo sazonal de distribuiçáo de precipitação bem característico, alternando uma estação seca de junho a novembro e uma estaçáo chuvosa de dezembro a maio. A média anual de precipitação é de $2.239 \mathrm{~mm}$, com $66,1 \%$ da precipitação distribuída na estação chuvosa e 33,9\% na estação seca. As temperaturas médias do ar variam de 25,2 ${ }^{\circ} \mathrm{C}$ a $26,2{ }^{\circ} \mathrm{C}$ por ano. Os solos predominantes na área da Província Petrolífera de Urucu são os Cambissolos e Argissolos, ocorrendo também algumas áreas com Latossolos e Gleissolos (Teixeira et al. 2006). A região é basicamente composta por dois grandes grupos de vegetaçấo: Floresta Ombrófila Densa de Terra-Firme (representando 80 a $85 \%$ da cobertura vegetal) e Floresta Ombrófila densa de várzea. A vegetação é composta por palmeiras, arvoretas, ervas e um grande número de árvores altas e finas, troncos retos e copas globosas (Amaral 1996).

\section{COLETA DOS DADOS}

Foram realizadas quatro expediçóes nos períodos de $02 \mathrm{a}$ 21 de julho de 2003, 04 a 15 de novembro de 2004, 02 a 16 de janeiro de 2007 e 02 a 14 de abril de 2007, totalizando 51 dias de coleta. Foram amostradas 42 clareiras antropizadas e a mata adjacente a cada clareira, ao longo de $50 \mathrm{~km}$ de malha viária da Base até o Porto Evandro (Figura 1).

Para obter uma amostragem mais representativa da regiáo, foram utilizados quatro métodos de coletas, que são considerados complementares: armadilha de interceptação e queda, procura limitada por tempo, a pé e de carro e encontros ocasionais (Prudente e Santos-Costa 2005). O material coletado, listado abaixo, foi depositado na Coleção Herpetológica do Museu Paraense Emílio Goeldi.

\section{MATERIAL COLETADO}

Aniliidae: Anilius scytale: MPEG 22197, MPEG 22198; Boidae: Boa constrictor: MPEG 21156, MPEG 22199; Corallus caninus: MPEG 22200; Corallus hortulanus: MPEG 20691, MPEG 20692, MPEG 22201, MPEG 22202, MPEG 22203, MPEG 22204; Epicrates cenchria: MPEG 20682; Colubridae: Chironius exoletus: MPEG 22206; Chironius fuscus: MPEG 21149, MPEG 21687, MPEG 21688, MPEG 22207, MPEG 22208, MPEG 22209; Chironius multiventris: MPEG 22210, MPEG 22211, MPEG 20704, MPEG 22212, MPEG 22213; Chironius scurrulus: MPEG 20683, MPEG 22214, MPEG 22215; Dendrophidion dendrophis: MPEG 22217, MPEG 22218, MPEG 22219; Leptophis ahaetulla: MPEG 20707, MPEG 21147; Oxybelis fulgidus: MPEG 20698, MPEG 20699, MPEG 20700, MPEG 20701, MPEG 20702, MPEG 20703, MPEG 22247; Pseustes poecilonotus: MPEG 20687, MPEG 21148, MPEG 22262; Pseustes sulphureus: MPEG 21154; Rhinobothryum lentiginosum: MPEG 20709, MPEG 22257, MPEG 22258; Spilotes pullatus: MPEG 22263, MPEG 22264, MPEG 22265; Tantilla melanocephala: MPEG 20708, MPEG 22267. MPEG 22268. Dipsadidae: Atractus alphosehogei: MPEG 21144; Atractus latifrons: MPEG 19261, MPEG 21690; Atractus torquatus: MPEG 21143, MPEG 22205; Atractus sp.: MPEG 21157; Clelia clelia: MPEG 22222; Dipsas catesbyi: MPEG 22220, MPEG 22221; Drepanoides anomalus: MPEG 22223; Helicops angulatus: MPEG 20688, MPEG 22224; Hydrops martii: MPEG 22225; Hydrops triangularis: MPEG 22226; Imantodes cenchoa: MPEG 20689, MPEG 21152, MPEG 21153, MPEG 22227; Imantodes lentiferus: MPEG 22228; Leptodeira annulata: MPEG 20685, MPEG 20705, 


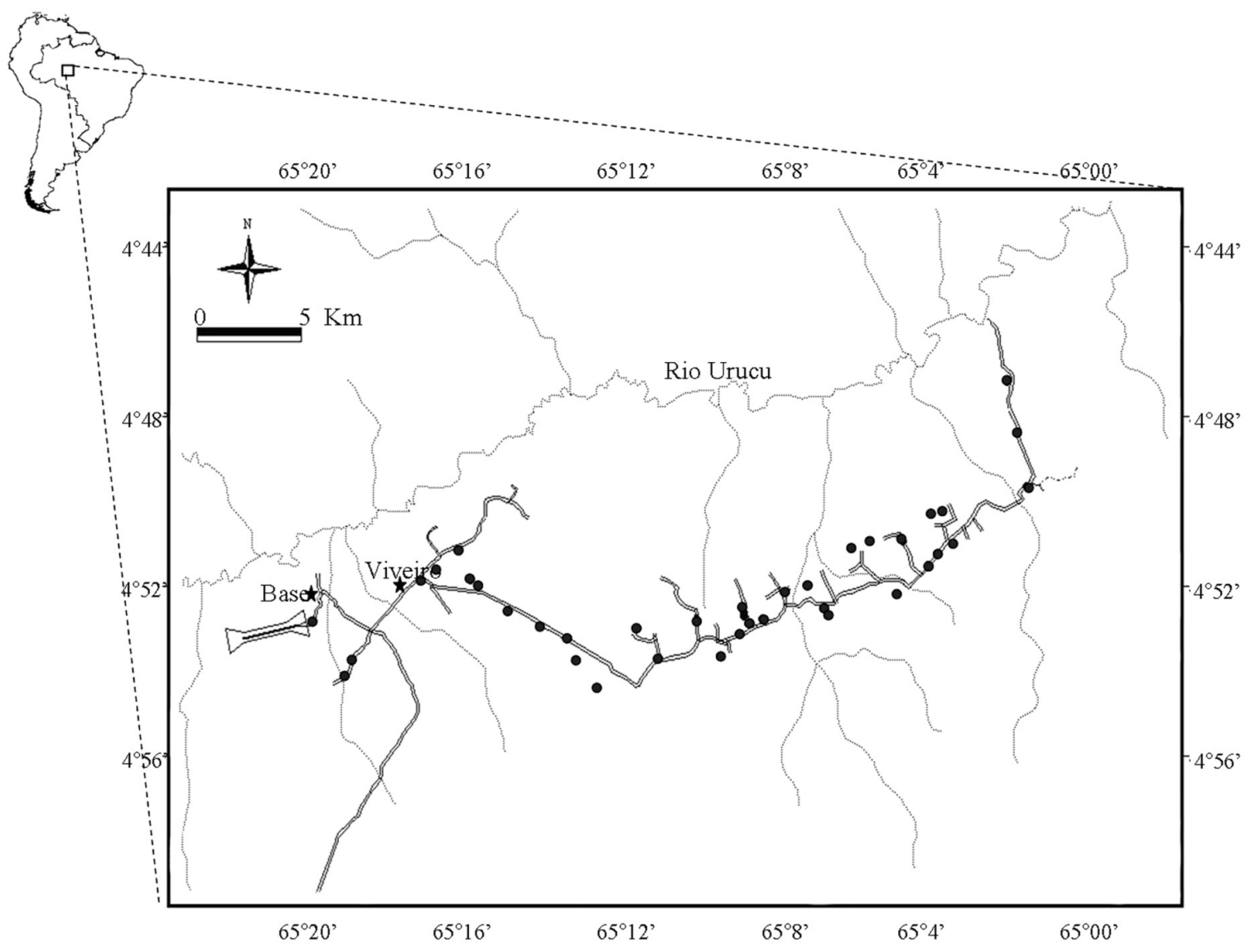

Figura 1 - Mapa de localização da Base Operacional Geólogo Pedro de Moura, Município de Coari, Amazonas, Brasil. Círculo fechado: pontos de coleta de serpentes

MPEG 22229, MPEG 22230; Liophis reginae: MPEG 21151, MPEG 22231, MPEG 22232, MPEG 22233, MPEG 22234, MPEG 22235, MPEG 22236, MPEG 22237, MPEG 22238, MPEG 22239, MPEG 22240, MPEG 22241, MPEG 22279, MPEG 22280; Liophis typhlus: MPEG 21150, MPEG 22242, MPEG 22243, MPEG 22244, MPEG 22245, MPEG 22246; Oxyrhopus petola: MPEG 21159, MPEG 22248, MPEG 22249; Oxyrhopus formosus: MPEG 20609, MPEG 20686, MPEG 20693, MPEG 20695, MPEG 20711, MPEG 22250; Philodryas viridissima: MPEG 20684, MPEG 20696, MPEG 20697, MPEG 21158, MPEG 22251, MPEG 22252, MPEG 22253, MPEG 22254, MPEG 22255; Pseudoboa coronata: MPEG 22256; Siphlophis compressus: MPEG 21155, MPEG 22259, MPEG 22260, MPEG 22261; Taeniophalus occipitalis: 22266; Xenodon rabdocephalus: MPEG 22269, MPEG 22270; Xenodon severus: MPEG 22216, MPEG 22271; Xenopholis scalaris: MPEG 22273; Philodryas boulengeri: MPEG 20680, MPEG 20681, MPEG 20706, MPEG 20694, MPEG 21261, MPEG 21260, MPEG 22273; Elapidae: Micrurus langsdorff: MPEG 18235; Micrurus spixii: MPEG 22274, MPEG 22275; Typhlopidae: Typhlops brongersmianus: MPEG 21145, MPEG
21146; Viperidae: Bothrops atrox: MPEG 22276, MPEG 22277, MPEG 22278; Lachesis muta: MPEG 20710.

\section{RESULTADOS E DISCUSSÃO}

Considerando os quatro métodos de coleta empregados, foram capturados 140 espécimes de serpentes na BOGPM distribuídos em 47 espécies, 33 gêneros e sete famílias: Typhlopidae (1 espécie), Aniliidae (1 espécie), Boidae (4 espécies), Colubridae (12 espécies), Dipsadidae (sensu Zaher et al. 2009) (25 espécies), Viperidae (2 espécies) e Elapidae (2 espécies) (Tabela 1$)$.

A procura limitada por tempo de carro foi o método que registrou o maior número de indivíduos vivos (ativos ou não) e mortos na área estudada (52,8\%). Embora os trabalhadores da BOGPM recebam treinamento para evitar atropelamentos de animais silvestres, o grande número de serpentes mortas na estrada, provavelmente esteja relacionado ao medo que esses animais despertam na maioria das pessoas.

A utilização de armadilhas de interceptação e queda em apenas uma expedição, provavelmente influenciou no baixo 
Tabela 1 - Lista de serpentes da Bacia Petrolífera de Urucu, Município de Coari, Amazonas, Brasil.

\begin{tabular}{|c|c|c|}
\hline Família & Espécie & $\mathrm{n}$ \\
\hline Typhlopidae & Typhlops brongersmianus Vanzolini, 1976 & 2 \\
\hline Aniliidae & Anilius scytale (Linnaeus, 1758) & 2 \\
\hline \multirow[t]{4}{*}{ Boidae } & Boa constrictor Linnaeus, 1758 & 2 \\
\hline & Corallus caninus (Linnaeus, 1758) & 1 \\
\hline & Corallus hortulanus (Linnaeus, 1758) & 6 \\
\hline & Epicrates cenchria (Linnaeus, 1758) & 1 \\
\hline \multirow[t]{12}{*}{ Colubridae } & Chironius exoletus (Linnaeus, 1758) & 1 \\
\hline & Chironius fuscus (Linnaeus, 1758) & 6 \\
\hline & Chironius multiventris Schmidt \& Walker, 1943 & 5 \\
\hline & Chironius scurrulus (Wagler, 1824) & 3 \\
\hline & Dendrophidion dendrophis (Schlegel, 1837) & 3 \\
\hline & Leptophis ahaetulla (Linnaeus, 1758) & 2 \\
\hline & Oxybelis fulgidus (Daudin, 1803) & 7 \\
\hline & Pseustes poecilonotus (Günther, 1858) & 3 \\
\hline & Pseustes sulphureus (Wagler, 1824) & 1 \\
\hline & Rhinobothryum lentiginosum (Scopoli, 1785) & 3 \\
\hline & Spilotes pullatus (Linnaeus, 1758) & 3 \\
\hline & Tantilla melanocephala (Linnaeus, 1758) & 3 \\
\hline \multirow[t]{20}{*}{ Dipsadidae } & Atractus sp. & 1 \\
\hline & Atractus alphonsehogei Cunha \& Nascimento, 1983 & 1 \\
\hline & Atractus latifrons (Günther, 1868) & 2 \\
\hline & Atractus torquatus (Duméril, Bibron \& Duméril, 1854) & 2 \\
\hline & Clelia clelia (Daudin, 1803) & 1 \\
\hline & Dipsas catesbyi (Sentzen, 1796) & 2 \\
\hline & Drepanoides anomalus (Jan, 1863) & 1 \\
\hline & Helicops angulatus (Linnaeus, 1758) & 2 \\
\hline & Hydrops martii (Wagler, 1824) & 1 \\
\hline & Hydrops triangularis (Wagler, 1824) & 1 \\
\hline & Imantodes cenchoa (Linnaeus, 1758) & 4 \\
\hline & Imantodes Ientiferus (Cope, 1894) & 1 \\
\hline & Leptodeira annulata (Linnaeus, 1758) & 4 \\
\hline & Liophis reginae (Linnaeus, 1758) & 14 \\
\hline & Liophis typhlus (Linnaeus, 1758) & 6 \\
\hline & Oxyrhopus formosus (Wied, 1820) & 6 \\
\hline & Oxyrhopus petola (Linnaeus, 1758) & 3 \\
\hline & Philodryas boulengeri (Procter, 1923) & 7 \\
\hline & Philodryas viridissima (Linnaeus, 1758) & 9 \\
\hline & Pseudoboa coronata Schneider, 1801 & 1 \\
\hline
\end{tabular}




\begin{tabular}{lcc}
\hline Tabela 1 - Continuação & & \\
\hline Família & Espécie & $n$ \\
\hline & Siphlophis compressus (Daudin, 1803) & 4 \\
& Taeniophallus occipitalis (Jan, 1863) & 2 \\
& Xenodon rabdocephalus (Weid, 1824) & 1 \\
\hline Elapidae & Xenodon severus (Linnaeus, 1758) & 2 \\
\hline Viperidae & Xenopholis scalaris (Wucherer, 1861) & 1 \\
\hline & Micrurus spixii Wagler, 1824 & 3 \\
\hline Total & Micrurus langsdorffi Wagler, 1824 & 1 \\
\hline
\end{tabular}

número de espécies fossoriais e semi-fossoriais registradas para a área (Typhlops brongesmianus, Anilius scytale, Atractus alphonsehogei, A. latifrons, A. torquatus e Atractus sp.). Da mesma forma, as espécies aquáticas e semi-aquáticas (Helicops angulatus, Hydrops martii e Hydrops triangularis) foram subamostradas nesse estudo, com a ausência de método específico para acessar esses ambientes.

Das 47 espécies coletadas, Liophis reginae $(n=14)$, Philodryas viridissima $(\mathrm{n}=9)$, Philodryas boulengeri $(\mathrm{n}=7)$ e Oxybelis fulgidus $(\mathrm{n}=7)$ foram as mais abundantes em toda região (Tabela 1).

Liophis reginae apresenta atualmente quatro subespécies, sendo L. r. macrossomus e L. $r$. semilineatus registradas para o Brasil. Costa (2002) revalidou a espécie L. oligolepis anteriormente incluída na sinonímia de $L$. $r$. semilineatus e elevou esta última à categoria de espécie. A proposta de Costa (2002) por não ter valor taxonômico (dados não publicados), seguiu-se a proposta de Cunha e Nascimento (1993), sendo considerado para a área estudo o táxon $L$. reginae.

A segunda espécie mais comum na região, Philodryas viridissima, é frequentemente registrada em áreas alteradas, apresentando uma grande plasticidade para viver em diversos tipos de ambiente natural ou antrópico. Esta capacidade pode, segundo Cunha e Nascimento (1993), estar associada à disponibilidade de presas que utilizam locais antropizada com área de vida, como pequenos roedores, anfíbios e aves, animais comumente encontrados nas clareiras da Base.

O registro de Philodryas boulengeri é considerado raro em coleçóes científicas, com a presença de apenas oito espécimes na área de influência da Bacia do Rio Madeira, incluindo o sudeste do Peru, Bolívia e alto Rio Amazonas no Brasil (Keiser 1974). Recentemente, Prudente et al. (2008) registraram esta espécie para os estados do Acre, Amazonas e Rondônia, e a consideraram comum para as regióes florestais da Amazônia Ocidental.

Devido a grande dificuldade de identificação, uma das espécies de Atractus registrada para área não foi identificada, sendo necessário uma análise mais refinada e com um maior número de exemplares. Esta dificuldade de identificação está relacionada ao grande número de espécies válidas (mais de 120 espécies), à grande semelhança morfológica entre as espécies, à ampla distribuição geográfica do gênero (do oeste do Panamá através das cadeias dos Andes da Venezuela até a Bolívia, Guianas, Floresta Amazônica, central e sudoeste do Brasil e norte da Argentina) e a distribuição restrita de algumas espécies (Peters e Orejas-Miranda 1970; Giraudo e Scrocchi 2000; Hoogmoed e Prudente 2003; Myers 2003; Myers e Schargel 2006; Prudente e Santos-Costa 2006).

Segundo Silva e Sites (1995) as localidades ao sul do Rio Amazonas, como Usina Hidrelétrica de Tucuruí (72 espécies), região Leste do Pará (90 espécies) e Usina Hidrelétrica de Samuel (92 espécies), são mais ricas quando comparadas com regiōes ao norte do Amazonas (Município de Manaus com 83 espécies; Reserva do INPA - WWF com 61 espécies e Usina Hidrelétrica de Balbina com 69 espécies). Para a região de Urucu, Montag et al. (2009) publicaram uma lista de fauna geral, onde constam 39 espécies de serpentes.

Os resultados obtidos nesse estudo mostram um incremento de sete espécies de serpentes para a região (Leptophis ahaetulla, Clelia clelia, Imantodes lentiferus, Taeniophallus occipitalis, Xenodon severus, Micrurus langsdorff e Corallus caninus) em relação à lista preliminar apresentada por Montag et al. (2009). Esses resultados evidenciam a necessidade de um maior esforço de coleta para que novos 
registros sejam adicinados para a área, a qual se encontra, muito provavelmente, subamostrada, se compararmos com os dados apresentados por Silva e Sites (1995).

\section{AGRADECIMENTOS}

Agradecemos ao Museu Paraense Emílio Goeldi, Universidade Federal do Pará, FINEP e PETROBRÁS pelo apoio na realização deste projeto; ao IBAMA (processo 02018.005457/01-36) pela autorização de coleta; ao Raimundo Rodrigues e Reginaldo Rocha pelo apoio técnico durante as expediçōes de campo em Urucu. A. L. C. Prudente agradece ao CNPq para Bolsa de PQ (processos 307999/2008-4).

\section{BIBLIOGRAFIA CITADA}

Amaral, I.L. 1996. Floristic diversity in upland forest, in Urucu region - AM. Dissertaçáo de Mestrado, Instituto Nacional de Pesquisas da AmazônialFundação Universidade do Amazonas. Manaus, Amazonas.104 pp (in Portuguese).

Ávila-Pires, T.C.S.; Hoogmoed, M.S.; Vitt, L.J. 2007. Herpetofauna in the Amazon, p. 13-43. In: Nascimento, L.B.; Oliveira, M.E. (Eds.) Herpetology in the Brazill II. Sociedade Brasileira de Herpetologia, Belo Horizonte, Minas Gerais (in Portuguese).

Cadle, J.E.; Greene, H.W. 1993. Phylogenetic patterns, biogeography, and ecological structure of Neotropical Snake assemblage, p. 281293. In: Ricklefs, R.E.; Schuluter, D. (Eds.). Species Diversity in Ecological Communities- Historical and geographical perpectives. University of Chicago Press, Chicago and London.

Costa, R.G.N. 2002. Morphological characterization of Liophis reginae semilineatus (Wagler, 1824) and Liophis reginae macrosomus (Amaral, 1935), and the taxonomic status of Liophis oligolepis Boulenger, 1905. Dissertação de Mestrado, Museu Paraense Emílio Goeldi/Univesidade Federal do Pará, Belém, Pará.77 pp (in Portuguese).

Cracraft, J. 1985. Historical biogeography and patterns or differentiation within the south American avifauna: areas of endemism, p. 49-84. In. Buley, A.; Foster, M.A.; Morton, E.S.; Ridgely, R.S.; BucKley, F.G.(Eds). Neotropical Ornitology. Vol. 36. Ornitology Monograph, McLean, Virginia, USA.

Cunha, O.R.; Nascimento F. P. 1993. Snakes of the Amazon. The snakes of eastern Pará. Boletim do Museu Paraense Emílio Goeldi, Zoologia, 9:1-191 (in Portuguese).

Giraudo, A. R.; Scrocchi, G. J. 2000. The genus Atractus (Serpentes: Colubridae) in Northeastern Argentina. Herpetological Journal, 10:81-90.

Hoogmoed, M.S., Prudente, A.L.C. 2003. A new species of Atractus (Reptilia: Ophidia: Colubridae: Dipsadinae) from the Amazon forest region in Brazil. Zoologische Mededelingen, 77:425-439.

Keiser Jr., E.D. 1974. A systematic study of the neotropical vine snake Oxybelis aeneus (Wagler). Bulletin of the Texas Memorial Museum, 51.

Martins, M.; Oliveira, M. E. 1998. Natural history of snakes in forest of the Manaus region, Central Amazonia, Brazil. Herpetological Natural History, 6: $78-150$.
Montag, L.F.A.; Prudente, A.LC.; Martins, M.; Aleixo, A.; Aamaral, I.L.; Bastos, N.C.; Bonaldo, A.; Candiani, D.F.; Filho, F.S.C.; Costa, C.E.C.; Persio, M.; Dias, S.C.; Espósito, M.C.; Feitosa, D.T.; Fernandes, J.A.M.; Furtado, I.S.; Gurgel, E.S.C.; LomanHung, N.F.; Lima-Filho, D.A.; Maschio, G. F.; Matos, F.D.A.; Mendonça, M.B.; Oliveira, I.R.P.; Oliveira, R.F.M.; Penha, J.A.N.; Poletto, F.; Rendeiro, A.C.; Rocha, A.E.S.; Silva, J.U.M.; Santos-Costa, M.C.; Silva e Silva, A.E.; Soares, M.L.; Wosiacki, W. List of species - Herpetofauna, p. 156-189. In: Prudente, A.L.; Rosário, D.P.; Montag, L.F.A., Monnerat, M.C.; Martins, M.B.; Cabral, P.R.; Lima, S.O. F. (Org.) (in Portuguese).

Myers, C.W. 2003. Rare snakes - Five New Species from Eastern Panama: Reviews of Northern Atractus and Southern Geophis (Colubridae: Dipsadinae). American Museum Novitates, 3391:1-47.

Myers, C.W.; Schargel, W.E. 2006. Morphological extremes-two new snakes of the genus Atractus from northwestern South America (Colubridae: Dipsadinae). American Museum Novitates, 3532:1-13.

Peters, J.A.; Orejas-Miranda, B. 1970. Catalogue of the Neotropical Squamata: Part I. Snakes. U. S. National Museum Bulletim, 297:1-347.

Prudente, A.L.C.; Santos-Costa, M.C. 2005. Checklist of snakes from Ferreira Penna Scientific Station, Eastern Amazonia, Pará state, Brazil. Boletim do Museu Paraense Emílio Goeldi. Série Ciências Naturais, 1(3): 203-208.

Prudente, A.L.C.; Santos-Costa, M.C. 2006. A new species of Atractus Wagler, 1828 (Serpentes: Colubridae) from eastern Amazonia, Brazil. Zootaxa, 1285:21-29.

Prudente, A.L.C.; Silva, M.A.A; Rocha, W.A.; Franco, F.L. 2008. Morphological variation in Xenoxybelis boulengeri (Procter, 1923) (Serpentes, Xenodontinae, Philodryadini). Zootaxa, 1743:53-61.

Silva Jr, N. J. da; Sites, Jr., J.W. 1995. Patterns of Diversity of Neotropical Squamate Reptile Species with Emphasis on the Brazilian Amazon and the Conservation Potential of Indigenous Reserves. Conservation Biology, 9(4): 873-901.

Teixeira, W.G.; Cruz, M.E.G.; Martins, G.C.; Macedo, R.S.; Correia, M.R.; Lima, H.N.; Rodrigues, M.R.L. Survey of pedology bases available to the Coari municipality - AM. In: II Workshop de avaliação técnica e científica - Rede CT-PETRO Amazônia, 2006. CD-ROM. (in Portuguese).

Vitt, L.J.; Vangilder, L.D. 1983. Ecology of snake community in the northeastern Brazil. Amphibia-Reptilia, 4: 273-296.

Zaher, H; Grazziotin, F.G.; Murphy, R.W.; Moura-Leite, J.C.; Bonatto, S.L. 2009. Molecular phylogeny of advanced snakes (Serpentes, Caenophidia) with an emphasis on South American Xenodontines: a revised classification and descriptions of new taxa. Papéis Avulsos de Zoologia, 49(11): 115-153.

Recebido em 11/03/2008

Aceito em 09/10/2009 